\title{
ERD as an index of anticipatory attention? Effects of stimulus degradation
}

\author{
MARCEL C.M. BASTIAANSEN, ${ }^{\mathrm{a}, \mathrm{b}}$ KOEN B.E. BÖCKER, ${ }^{\mathrm{c}}$ AND CORNELIS H.M. BRUNIA ${ }^{\mathrm{b}}$ \\ ${ }^{a}$ Cooperation Centre Tilburg and Eindhoven Universities, Tilburg, the Netherlands \\ ${ }^{\mathrm{b}}$ Psychonomics Section, Tilburg University, the Netherlands \\ ${ }^{\mathrm{c}}$ Department of Psychopharmacology, Utrecht University, the Netherlands
}

\begin{abstract}
Previous research has suggested that the stimulus-preceding negativity (SPN) is largely independent of stimulus modality. In contrast, the scalp topography of the event-related desynchronization (ERD) related to the anticipation of stimuli providing knowledge of results (KR) is modality dependent. These findings, combined with functional SPN research, lead to the hypothesis that anticipatory ERD reflects anticipatory attention, whereas the SPN mainly depends on the affective-motivational properties of the anticipated stimulus. To further investigate the prestimulus ERD, and compare this measure with the SPN, 12 participants performed a time-estimation task, and were informed about the quality of their time estimation by an auditory or a visual stimulus providing KR. The KR stimuli could be either intact or degraded.

Auditory degraded KR stimuli were less effective than other KR stimuli in guiding subsequent behavior, and were preceded by a larger SPN. There were no effects of degradation on the SPN in the visual modality. Preceding auditory KR stimuli no ERD was present, whereas preceding visual stimuli an occipital ERD was found. However, contrary to expectation, the latter was larger preceding intact than preceding degraded stimuli. It is concluded that the data largely agree with an interpretation of the pre-KR SPN as a reflection of the anticipation of the affective-motivational value of KR stimuli, and of the prestimulus ERD as a perceptual anticipatory attention process.
\end{abstract}

Descriptors: SPN, Slow potentials, Event-related desynchronization, Stimulus degradation, Anticipatory attention, Affective-motivational

Anticipatory behavior is directed at a predictable, upcoming event. It serves the goal of a faster or more efficient information processing than would have been possible without anticipation. Elsewhere, we have proposed that anticipatory behavior is realized through a thalamocortical gating mechanism (see, e.g., Bastiaansen, Brunia, \& Böcker, 1999, and Brunia, 1993, 1999, for detailed descriptions of the proposed neurophysiological model).

The present article focuses on the sensory aspect of anticipatory behavior, that is, the anticipatory attention for an upcoming stimulus. Our neurophysiological model for anticipatory behavior predicts that anticipatory attention manifests itself as a cortical activation preceding the presentation of stimuli, which is restricted to the cortical area corresponding to the modality of the stimulus. Two EEG measures might be appropriate for revealing manifestations of anticipatory attention: (1) negative slow potentials, which stem from a predominance of excitatory postsynaptic potentials at the dendrites of cortical neurons, leading to a subthreshold depolarization of these cells and a concurrent increase in the readiness of

The authors wish to thank G. Pfurtscheller for providing the software used for part of the data analyses, and G.J.M. Van Boxtel for valuable comments on an earlier version of this article.

Address reprint requests to: M.C.M. Bastiaansen, Max Planck Institute for Psycholinguistics, P.O. Box 310, 6500 AH Nijmegen, the Netherlands. E-mail marcel.bastiaansen@mpi.nl. these cells to fire (Birbaumer, Elbert, Canavan, \& Rockstroh, 1990); (2) desynchronization of the EEG over the activated cortex, reflecting a disruption of synchronized activity in functionally related groups of cortical neurons (Steriade, Gloor, Llinás, Lopes da Silva, \& Mesulam, 1990; Lopes da Silva, 1991). This can be seen as a transition of cortical idling to cortical activity.

In the last two decades, much attention has been devoted in our laboratory to identifying negative slow potential shifts related to the anticipation of sensory input. To this end, a paradigm has been developed in which subjects are asked to perform a time-estimation task, and are subsequently informed about the quality of their time estimation by a stimulus providing knowledge of results (KR; Damen \& Brunia, 1987). Preceding the KR stimulus, a negative slow potential can be recorded that has been termed the stimuluspreceding negativity (SPN). Subsequent research has been aimed at clarifying the functional significance and neuroanatomical substrates of the SPN (see Böcker \& Van Boxtel, 1997, and Van Boxtel, 1994, for reviews). If the SPN is a purely perceptual anticipatory process, one would expect the SPN (1) to be independent of the type of information conveyed by the anticipated stimulus, and (2) to be maximal, or at least clearly distinguishable over the sensory cortex corresponding to the modality of the stimulus.

Ad (1). The SPN has been found prior to three types of stimuli: KR stimuli (e.g., Böcker, Brunia, \& Van Den Berg-Lenssen, 1994; Brunia \& Damen, 1988; Chwilla \& Brunia, 1991a; Damen \& 
Brunia, 1987, 1994; Grünewald, Grünewald-Zuberbier, Hömberg, \& Schuhmacher, 1984); instruction stimuli, transmitting information about a future task (e.g., Damen \& Brunia, 1994; Gaillard \& Van Beijsterveld, 1991; Rösler, 1991; Van Boxtel, 1994; Van Boxtel \& Brunia, 1994); and probe stimuli, with which the outcome of a previous task has to be matched (e.g., Chwilla \& Brunia, 1991b, 1992; Ruchkin, Johnson, Mahaffey, \& Sutton 1988). Together these studies revealed that both the amplitude and the lateralization of the SPN vary with the type of stimulus that is anticipated.

Ad (2). The SPN has been recorded prior to auditory and visual stimuli. However, only two studies have directly compared its scalp distribution as a function of stimulus modality in a withinsubjects design. The first study, by Grünewald et al. (1984, experiment B), did not include electrodes over the relevant sensory cortices, which severely limits the value of the results as far as scalp topography is concerned. The second study, by Böcker et al. (1994) used a larger set of electrodes (i.e., 23) approximately equally distributed over the entire scalp. Although the SPN was larger preceding auditory stimuli, there was a striking similarity in scalp topography of the SPN for the two modalities: Both had a frontotemporal maximum. Thus, the Böcker et al. study does not support the hypothesis that the SPN is maximal, or has a clearly distinguishable component, over the sensory cortex corresponding to the modality of the anticipated stimulus.

The available experimental evidence does not support an interpretation of the SPN in terms of (perceptual) anticipatory attention for an upcoming stimulus. This leaves the question of what kind of process is reflected by the SPN. Because the SPN differs with the type of stimulus anticipated, it appears reasonable to hypothesize that the SPN at least partially reflects the presetting of cortical areas specifically related to the execution of the task at hand rather than the presetting of the sensory cortex (although arguments for the latter have recently been presented by Brunia and Van Boxtel, in press). This suggests that the SPN is not a unitary phenomenon. If we consider the types of stimuli that evoke an SPN, two of the three stimulus types (KR stimuli and probe stimuli) evaluate the past performance of the subject. Therefore, it could be argued that these two instances of "the" SPN reflect the anticipation of affectivemotivational stimuli. Support for this interpretation comes from three studies. First, Kotani, Hiraki, and Aihara (1999) performed an EEG study using the time estimation + KR paradigm, in which they manipulated the motivational and the affective value of the KR stimulus independently. Higher motivation led to larger SPN amplitudes, whereas the affective manipulation did not influence SPN amplitude. Second, Böcker et al. (1994) showed that regardless of stimulus modality, a large part of the pre-KR SPN could be explained by a bilateral pair of frontotemporal dipoles that probably represent activity of the Insulae Reili. This is a bilateral cortical structure buried within the Sylvian fissure, which is assumed to be involved in the affective-motivational coloring of stimuli (Mesulam \& Mufson, 1985). Third, Brunia, De Jong, Van Den Berg-Lenssen, and Paans (2000) performed a PET study using the time estimation + KR paradigm, and also found activation of the posterior part of the Insulae Reili, therewith supporting the results of the dipole modeling study of Böcker et al. Together, these three studies suggest that the SPN preceding stimuli that evaluate past performance (such as KR stimuli, or probe stimuli with which the outcome of a previous task has to be matched) reflects the anticipation of the affective-motivational aspects of the stimulus rather than perceptual anticipatory attention.
Another candidate for identifying an activation of the sensory cortex during anticipatory attention for an upcoming stimulus is EEG desynchronization, as we mentioned above. EEG desynchronization can be quantified by a technique known as event-related desynchronization (ERD; Pfurtscheller \& Aranibar, 1977). This technique may be more appropriate to study anticipatory attention, because the same interactions between the thalamic reticular nucleus, the thalamocortical relay nuclei, and the cortex that are thought to underlie the mechanism of anticipatory attention (Brunia, 1999) also form the neural basis of ERD measures (e.g., Lopes da Silva \& Pfurtscheller, 1999; Steriade et al., 1990). To investigate this, the SPN data of the Böcker et al. (1994) study were reanalyzed with the ERD technique (Bastiaansen, Böcker, Cluitmans, \& Brunia, 1999). The results showed an ERD preceding visual KR stimuli that was maximal over occipital electrode positions, whereas preceding auditory KR stimuli, no appreciable ERD was found.

A possible explanation of the absence of the expected bilateral temporal ERD preceding auditory stimuli might be that rhythmic activity from the auditory cortex cannot be measured with scalprecorded EEG, as has been demonstrated by Niedermeyer (1990, 1991; see also Niedermeyer, 1997, for a review). Instead, with MEG it is possible to record such a rhythm, which has been termed the tau rhythm (Lehtelä, Salmelin, \& Hari, 1997; Tiihonen et al., 1991; see Hari, Salmelin, Mäkelä, Salenius, \& Helle, 1997, for a review). If this was the reason for the absence of an ERD over the auditory cortex preceding the auditory KR, then this effect should be present in MEG data. This idea was partially confirmed in a subsequent study (Bastiaansen, Böcker, Brunia, de Munck, \& Speckreijse, 2001): In two out of the five subjects studied, we found a bilateral temporal ERD preceding auditory KR stimuli in the MEG that was not present in the EEG.

The main purpose of the present article is to further elucidate the functional significance of the prestimulus ERD. We feel that if we can influence the magnitude and/or scalp topography of the prestimulus ERD by varying stimulus parameters, the interpretation of this phenomenon in terms of anticipatory attention would gain strength. To this end, we conducted an experiment in which subjects were asked to perform a time-estimation task, and were subsequently informed about the quality of their time estimation by a KR stimulus. The KR stimulus could be either auditory or visual, and either intact or perceptually degraded.

We argued that stimulus degradation might have two effects. First, it asks for a more efficient stimulus processing to extract the relevant information from the KR stimulus. This implies that perceptual anticipatory attention should be more prominent preceding degraded than preceding intact KR stimuli. If the prestimulus ERD we have previously reported is a true index of perceptual anticipatory attention, one would expect the (compensatory) increase in perceptual anticipatory attention called for by stimulus degradation to be reflected in an increase in this index. Assuming that the SPN reflects anticipation of the affective/motivational properties of the KR stimulus, we would not however expect influences of perceptual difficulty on the SPN. Second, stimulus degradation may lead to an increase in task difficulty if the degree of degradation is such that the stimuli are not always recognized. At the behavioral level, this should be reflected in a decrease in task performance, and/or a decrease in the effectiveness of the KR stimulus in guiding subsequent behavior (i.e., the time estimation on the next trial). Furthermore, an increase in task difficulty should affect the subject's motivation. If the SPN reflects the anticipation of the processing of the affective-motivational value of a stimulus, 
one would expect this to be reflected in an increase in the amplitude of the SPN (e.g., Chwilla \& Brunia, 1991a; Kotani et al., 1999). This effect on the SPN amplitude should be independent of stimulus modality.

To our knowledge, the effect of stimulus discriminability upon the prestimulus ERD has not been investigated yet. Furthermore, the effect of stimulus discriminability on the amplitude of the SPN has been addressed only by two studies (Chwilla \& Brunia, 1991b; Kotani \& Aihara, 1999) that yielded contradictory results. Assuming that the prestimulus ERD is a manifestation of a perceptually based anticipatory attention process, we expected the following effects to occur. (1) A larger prestimulus ERD preceding degraded KR stimuli than preceding intact KR stimuli. Based on the results of previous experiments however, we expect the prestimulus ERD to occur only in the visual modality. (2) No differences in scalp topography in the SPN preceding auditory and visual KR stimuli. (3) An increase in amplitude of the SPN preceding degraded KR stimuli compared to intact stimuli, provided that notion of increased task difficulty as a result of stimulus degradation is supported by the behavioral data. Finally, we will investigate the lower $(8-10 \mathrm{~Hz})$ and upper $(10-12 \mathrm{~Hz})$ alpha bands separately, to take into account the hypothesis formulated by Klimesch (1999) that the lower alpha band reflects alertness or expectancy whereas the upper alpha band reflects task-specific processes. It should be noted, however, that this claim is based on the results of two experiments (Klimesch, Doppelmayr, Russegger, Pachinger, \& Schweiger, 1998; Klimesch, Pfurtscheller, \& Schimke, 1992) in which a response was required to the stimuli that were anticipated. Because preparing for a response also elicits ERD in the alpha frequency range, it is unclear whether the lower alpha ERD in these experiments was produced by motor preparatory processes or by anticipatory attention for the upcoming stimulus.

\section{Methods}

\section{Subjects}

Twelve right-handed participants, 1 man and 11 women, aged $18-29(M=21, S D=3)$ participated in the experiment. All were undergraduate students. They either received course credit points or were paid a small fee (Dfl. 10.00, about US\$ 5.00 per hour).

\section{Design and Procedure}

Participants were seated in a dimly illuminated, sound-attenuating chamber. The experiment consisted of two tasks: a voluntarymovement task and a time-estimation task. In the voluntarymovement task, subjects were instructed to produce rapid selfpaced unilateral flexions of index finger and thumb at a slow pace (4-6 movements per minute), with either the left or the right hand. Trials were discarded from further analysis if the interresponse interval was shorter than $8.5 \mathrm{~s}$. On the average, $2.4 \%$ of the trials were rejected on these grounds. A total of 80 behaviorally correct trials were recorded for each response side.

In the time-estimation task, subjects were instructed to produce a rapid unilateral flexion of index finger and thumb, 4,000 ms after the onset of an auditory warning stimulus (WS; $1000 \mathrm{~Hz}, 70 \mathrm{~dB}(\mathrm{~A})$, $100 \mathrm{~ms})$. Two seconds after response onset, subjects were informed about the correctness of the estimated time interval by either an auditory or a visual KR stimulus, that could be either intact or degraded (see below). The KR stimulus indicated whether the response was too early, correct, or too late. During two initial training blocks (one with auditory KR and one with visual KR), the window defining a correct response was individually adjusted according to the algorithm described by Wetherill and Levitt (1965), so as to obtain about $60 \%$ correct responses. This window was used in the remainder of the experiment. The time-out was set at 4,750 ms after WS onset, and was followed by a KR corresponding to a response too late. Trials in which the estimated time interval was shorter than $3,500 \mathrm{~ms}$ or longer than $4,500 \mathrm{~ms}$ were discarded from further analysis. On the average, $4.7 \%$ of the trials were rejected on these grounds.

A total of 80 behaviorally correct trials was recorded for each condition. The order of the eight time-estimation conditions (2 response sides $\times 2 \mathrm{KR}$ modalities $\times 2$ stimulus types) was randomized over subjects. The two voluntary-movement conditions (one for each response side) always preceded the time estimation conditions, in order to prevent carryover effects from the timeestimation task to the voluntary movement task.

Responses were recorded by force transducers, mounted on both arms of the chair. The criterion force was defined as $20 \%$ of the participant's maximum voluntary force, which was determined prior to the experiment during a unilateral tonic contraction of $3 \mathrm{~s}$ duration, separately for the left and right hands. Response onset was defined as the moment the criterion force was reached.

\section{KR Stimuli}

Visual KR stimuli (duration $150 \mathrm{~ms}$ ) were presented on a 15 in. computer monitor located $1.2 \mathrm{~m}$ in front of the subject. The KR stimulus could be a horizontal bar (in case the estimated interval was too short), a vertical bar (for a correctly timed response), or a plus sign (indicating that the estimated time interval was too long). In the intact $\mathrm{KR}$ condition, the KR appeared as a white figure on a black background. In the degraded KR condition, random patterns of small squares were overlaid on the stimulus. Within each square, the colors of foreground and background were inverted. A total of 20 different random square patterns was used. Figure 1 presents examples of intact and degraded visual KR stimuli in all three response categories.

Auditory KR stimuli (70 dB(A), $150 \mathrm{~ms}$ ) were presented through a loudspeaker located $1.2 \mathrm{~m}$ in front of the subject, just underneath the computer monitor. In the intact KR condition, the KR stimuli were pure tones with a frequency of 2000,1000 , or $500 \mathrm{~Hz}$, corresponding to estimated intervals that were too short, correct, or too long, respectively. In the degraded KR condition, white noise was added to the tones, but the overall output amplitude was kept constant at $70 \mathrm{~dB}(\mathrm{~A})$.
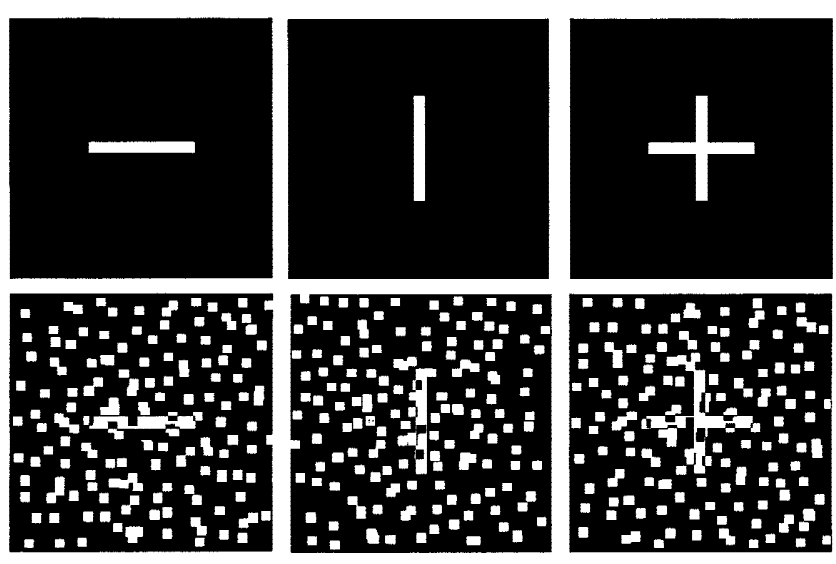

Figure 1. Examples of intact and degraded visual stimuli in all three response categories. 
In a behavioral pilot experiment, a three-choice reaction time task with 9 participants (who were not included in the actual experiment), we determined the level of stimulus degradation, that is, the amount of squares for visual stimuli and the ratio of the white noise to the tone for auditory stimuli. For each modality, we presented one block of 60 intact stimuli, and five blocks of 60 degraded stimuli differing in the level of degradation. The participants were asked to classify both the intact and the degraded stimuli by pressing that response button, out of the three response buttons mounted on the right arm of the chair, that corresponded to the KR stimulus. Based on a visual inspection of the classification results, for each modality, one level of stimulus degradation was selected in which the percentage of correct classifications was identical to that of the intact stimuli (that is, between 99.6 and $99.8 \%$ correct classifications). The reason for this is that we wanted to manipulate only perceptual difficulty, not task difficulty, in order to prevent a confounding of both processes. On these stimuli, the reaction time (RT) effects were tested by an ANOVA for repeated measures with variables modality (auditory, visual) and stimulus type (intact, degraded). This analysis yielded a main effect of modality, $F(1,8)=6.51, p=.0340$, indicating that RT was longer following visual $(738 \mathrm{~ms})$ than following auditory (605 ms) stimuli, and a main effect of degradation, $F(1,8)=5.96$, $p=.0405$, indicating that RT was longer in the degraded $(751 \mathrm{~ms})$ than in the intact $(592 \mathrm{~ms})$ condition. The absence of an interaction effect, $F(1,8)=0.692, p=.4300$, indicated that the RT effect of stimulus degradation was similar for both modalities.

\section{Electrophysiological Recordings}

For the EEG recordings, 27 nonpolarizing Beckman 8-mm Ag$\mathrm{AgCl}$ electrodes were affixed to the scalp, most of them placed according to the international 10-20 system. Standard positions were Fp1, Fp2, F7, F3, F4, F8, T3, Cz, T4, P3, Pz, and P4. Nonstandard positions were $\mathrm{C} 3^{\prime}$ and $\mathrm{C}^{\prime}$ ', which are $1 \mathrm{~cm}$ anterior to $\mathrm{C} 3$ and $\mathrm{C} 4$, respectively; TC3 and TC4, which are at one third of the distance from $\mathrm{T} 3$ to $\mathrm{C} 3$ and from $\mathrm{T} 4$ to $\mathrm{C} 4$, respectively; TP3 and TP4, which are in the center of T3, P3, T5, and C3 and in that of $\mathrm{T} 4, \mathrm{P} 4, \mathrm{~T} 6$, and $\mathrm{C} 4$, respectively; $\mathrm{O}^{\prime}, \mathrm{Oz}^{\prime}$, and $\mathrm{O} 2$ ', which are $1 \mathrm{~cm}$ anterior to $\mathrm{O} 1, \mathrm{Oz}$, and $\mathrm{O} 2$, respectively; and $\mathrm{O}^{\prime \prime}, \mathrm{Oz}^{\prime \prime}$, and $\mathrm{O} 2$ ", which are $2 \mathrm{~cm}$ posterior to $\mathrm{O} 1, \mathrm{Oz}$, and $\mathrm{O} 2$, respectively. Electrode impedance was kept below $5 \mathrm{k} \Omega$. Software-linked mastoids served as a reference. The EEG was amplified with a 30-s time constant, and a $70 \mathrm{~Hz}(-42 \mathrm{~dB} /$ octave $)$ low-pass filter. Epochs of 3,000 ms premovement to 3,000 ms postmovement were digitized with a sampling frequency of $256 \mathrm{~Hz}$.

The horizontal EOG from the outer canthi and the vertical EOGs of both eyes were recorded, and an off-line EOG correction was performed (Van den Berg-Lenssen, Brunia, \& Blom, 1989). Subsequently, a semiautomatic artifact detection was performed, discarding trials containing spikes and large drift.

\section{Data Reduction and Analysis}

Behavioral data. Two behavioral measures were derived from the RT data. First, the quality of time estimation was monitored by computing the percentages of too fast, correctly timed, or too slow responses. These data were analyzed by an ANOVA with KR modality (auditory, visual), KR type (intact, degraded), response side (left, right), and response category (too early, correct, too late) as repeated measures. Second, the effectiveness of the KR stimulus was assessed as follows: for trials with too early or too late responses, we computed the percentage of subsequent trials for which the RT changed in the desired direction (i.e., an RT de- crease following a trial with a too late response, and an RT increase following a trial with a too early response). These data were analyzed by an ANOVA with KR modality (auditory, visual), KR type (intact, degraded) and response side (left, right) as repeated measures.

Slow potentials. For the computation of the slow potentials, artifact-free trials, from $3,000 \mathrm{~ms}$ premovement to $3,000 \mathrm{~ms}$ postmovement were averaged, and the data were baseline-corrected from 2,750 to 2,000 ms prior to movement onset. The readiness potential (RP; Kornhuber \& Deecke, 1965) was quantified as the average amplitude in a 100-ms window preceding response onset. These data were subjected to an ANOVA with variables condition (voluntary movement, KR auditory intact, KR auditory degraded, KR visual intact, KR visual degraded), response side (left hand, right hand), electrode pair (frontal $(\mathrm{F} 3+\mathrm{F} 4)$, central $\left(\mathrm{C} 3^{\prime}+\mathrm{C} 4^{\prime}\right)$, temporal $(\mathrm{TC} 3+\mathrm{TC} 4)$, occipital $\left.\left(\mathrm{O}^{\prime}+\mathrm{O} 2^{\prime}\right)\right)$ and hemisphere (left, right).

The SPN (Damen \& Brunia, 1987) was quantified as the average amplitude in a 100 -ms window preceding the presentation of the KR stimulus. These data were subjected to an ANOVA with variables KR modality (auditory, visual), KR type (intact, degraded), response side (left hand, right hand), electrode pair (frontal $(\mathrm{F} 3+\mathrm{F} 4)$, central $\left(\mathrm{C}^{\prime}+\mathrm{C}^{\prime}\right)$, temporal $(\mathrm{TC} 3+\mathrm{TC} 4)$, occipital $\left.\left(\mathrm{O} 1^{\prime}+\mathrm{O} 2^{\prime}\right)\right)$ and hemisphere (left, right).

$E R D$. The ERD was computed as follows. First a weighted local average reference (Hjorth, 1975) was computed (the values at surrounding electrodes were weighted by the inverse of their distance to the target electrode), as is mostly done in ERD computation (e.g., Pfurtscheller, 1991). Next, the EEG was bandpass filtered using an FFT filter in two frequency bands: $8-10 \mathrm{~Hz}$ and 10-12 Hz. The data then were transformed into power values by squaring the amplitudes. Intervals of 64 consecutive samples were averaged, giving rise to 24 time intervals of $250 \mathrm{~ms}$ each. For each subject, data were averaged over trials, and ERD was computed as the percentage power increase (event-related synchronization, or ERS) or decrease (ERD) for a particular time interval in a particular frequency band, relative to the reference interval from 2,750 to 2,000 $\mathrm{ms}$ premovement. The end of this reference interval corresponds to the point in time when movement-related ERD starts (e.g., Pfurtscheller \& Berghold, 1989).

For the statistical analysis of the ERD, we were mainly interested in condition differences in the ERD immediately preceding the presentation of the KR stimulus. First however, we wanted to determine if differences between conditions in ERD can be attributed to differences in the level of alpha power in the reference interval. Therefore two ANOVAs were performed on the absolute power in the reference interval, one for the $8-10 \mathrm{~Hz}$ band and one for the $10-12 \mathrm{~Hz}$ band. For both ANOVAs, variables were condition (voluntary movement, KR auditory intact, KR auditory degraded, KR visual intact, KR visual degraded), response side (left hand, right hand), electrode pair (frontal $(\mathrm{F} 3+\mathrm{F} 4)$, central $(\mathrm{C} 3+$ $\mathrm{C} 4)$, temporal $(\mathrm{TC} 3+\mathrm{TC} 4)$, occipital $\left.\left(\mathrm{O}^{\prime}{ }^{\prime}+\mathrm{O} 2^{\prime}\right)\right)$, and hemisphere (left, right).

Next, single-sample $t$ tests were used to determine whether the ERD at temporal and occipital electrode positions in the last 250-ms interval preceding the KR stimulus significantly differs from zero, that is, whether there is a significant ERD over the auditory and visual cortices. To limit the number of $t$ tests, we averaged the ERD over response sides and hemispheres, because 
effects involving these factors were found to be caused exclusively by postmovement processes (see Results section).

Third, we wanted to test for differences between conditions in the prestimulus ERD by means of an ANOVA. On the basis of previous results however, we expected a postmovement ERS at central electrodes that lasts until after the presentation of the KR stimulus, and which interferes with the prestimulus ERD (Bastiaansen, Böcker, et al., 1999). In this previous study, we therefore subtracted the ERD in the voluntary movement condition from the ERD in the time estimation conditions, leaving the stimulusrelated ERD intact in the subtracted data. This approach proved to be a good way to solve the problem (see Bastiaansen, Böcker, et al., 1999). However, such a subtraction can be done reliably only if the movement-related ERD is similar in all conditions. To verify this, we first performed an ANOVA on the movement-related ERD. We used three time intervals to assess differences between conditions in the movement-related ERD: an early and a late premovement interval, respectively corresponding to intervals from 1,250 to $1,000 \mathrm{~ms}$ and from 250 to $0 \mathrm{~ms}$ prior to movement onset, and the last $250 \mathrm{~ms}$ interval preceding stimulus presentation. For this ANOVA, we used only the ERD at central (C3' and $\left.\mathrm{C} 4^{\prime}\right)$ channels, where movement-related processes are most prominent. Variables were frequency band $(8-10 \mathrm{~Hz}, 10-12 \mathrm{~Hz})$, time interval (1,250-1,000 ms premovement, 250-0 ms premovement, 250$0 \mathrm{~ms}$ prestimulus), condition (voluntary movement, KR auditory intact, KR auditory degraded, KR visual intact, KR visual degraded), response side (left hand, right hand), and hemisphere (left, right).

For the analysis of condition differences in the prestimulus ERD, from 250 to $0 \mathrm{~ms}$ prestimulus, we used only temporal and occipital electrode positions. The reason for this is that visual inspection of the ERD maps indicated that there were no appreciable ERD/ERS effects at frontal electrode positions, whereas at central positions there is a large postmovement ERS; therefore only the ERD at temporal and occipital channels is of interest. Variables were frequency band $(8-10 \mathrm{~Hz}, 10-12 \mathrm{~Hz}), \mathrm{KR}$ modality (auditory, visual), KR type (intact, degraded), response side (left hand, right hand), electrode pair (temporal (TC3 + TC4), occipital $\left.\left(\mathrm{O}^{\prime}+\mathrm{O} 2^{\prime}\right)\right)$ and hemisphere (left hemisphere, right hemisphere).

For all ANOVAs that were performed, degrees of freedom were corrected using the Greenhouse-Geisser epsilon ( $\epsilon$, Vasey \& Thayer, 1987) when necessary, and significant interactions were clarified by breaking them down into simple effects.

\section{Results}

\section{Behavioral data}

Over subjects, the average window defining correct responses was $525 \pm 9 \mathrm{~ms}$ (throughout the results section, $M \pm S E M$ will be reported). With respect to the quality of the time estimation, the ANOVA on the percentages too early, correct, and too late responses reveals that subjects produce more correct responses $(57 \pm$ $1 \%)$ than too early or too late responses $(20 \pm 2 \%$ and $23 \pm 1 \%$, respectively; main effect of response category: $F(2,10)=61.48$, $p<.0000, \epsilon=.9859)$. Furthermore, in conditions with auditory KR stimuli, subjects performed poorer than in conditions with visual KR stimuli, as indicated by an interaction of KR modality with response category, $F(2,10)=9.62, p=.0024, \epsilon=.88094$. In the conditions with auditory KR, the distribution was $22 \pm 2 \%$ too early, $54 \pm 2 \%$ correct, and $24 \pm 2 \%$ too late responses, whereas in the visual KR conditions, these percentages were $18 \pm 1 \%, 60 \pm$ $1 \%$, and $22 \pm 1 \%$, respectively.
KR stimuli indicating that a response was too early or too late resulted, on the subsequent trial, in an average of $86 \pm 1 \%$ of the cases in an adjustment of the RT in the desired direction. An interaction of $\mathrm{KR}$ modality with $\mathrm{KR}$ type, $F(1,11)=7.37, p=$ .0201 , revealed that in the auditory modality, stimulus degradation affected the effectiveness of the KR stimulus $(83 \pm 2 \%$ vs. $88 \pm$ $1 \%$ correct adjustments in the degraded and intact conditions, respectively, $F(1,11)=9.66, p=.0100$. This is not the case in the visual modality $(86 \pm 1 \%$ vs. $87 \pm 2 \%$ correct adjustments in the intact and degraded conditions, respectively; $F(1,11)=0.58, p=$ .4612).

\section{Slow potentials}

Figure 2 presents the time courses of the slow potentials recorded during the voluntary-movement tasks and the time-estimation tasks, at selected channels. Preceding the movement, a slow negative potential shift can be observed, which we identified as a readiness potential (Kornhuber \& Deecke, 1965). Preceding the presentation of the KR stimulus, a slow negative potential is present that we identified as a SPN (Damen \& Brunia, 1987). Figure 3 presents topographic maps of the average SPN in the last 100-ms interval preceding stimulus presentation.

Readiness Potential. The ANOVA revealed that the RP has a central maximum (main effect of electrode pair: $F(3,33)=12.19$, $p=.0006, \epsilon=.59$ ) and a contralateral dominance (interaction of response side and hemisphere: $F(1,11)=48.94, p<.0001$; simple effect of hemisphere for left- and right-hand movements: $F(1,11)=$ $21.71, p=.0007$, and $F(1,11)=13.75, p=.0035$, respectively). Moreover, this contralateral dominance is present at frontal, central, and temporal sites, not at occipital electrode positions (interaction of response side with electrode pair and hemisphere: $F(3,33)=51.45, p<.0001)$. The ANOVA did not reveal any differences between conditions in the RP.

Stimulus-Preceding Negativity. The results of the ANOVA on the SPN amplitudes are summarized in Table 1. The main effect of Electrode Pair indicated that the SPN was maximal at temporal positions. The SPN was larger preceding degraded $(-0.62 \pm$ $0.2 \mu \mathrm{V})$ than preceding intact $(+0.28 \pm 0.2 \mu \mathrm{V}) \mathrm{KR}$ stimuli (main effect of KR type). However, this amplitude enhancement as a result of stimulus degradation was only present preceding auditory KR stimuli, as indicated by a marginally significant interaction of KR modality with KR type (cf. Table 1). The underlying simple effects clearly support this interaction (simple effect of KR type in the auditory modality: $F(1,11)=7.75, p=.0178$; simple effect of KR type in the visual modality: $F(1,11)=0.09, p=.7721$ ). Furthermore, the interaction Modality $\times$ KR Type $\times$ Hemisphere revealed that the amplitude enhancement of the SPN as a result of stimulus degradation in the auditory modality was largest over the left hemisphere. This can also be seen in Table 2, which summarizes the SPN amplitudes at selected electrodes.

Finally, the SPN was largest over the hemisphere contralateral to the response side (interaction of response side with hemisphere), but this effect was significant only at central and temporal channels (interaction $\mathrm{R} \times \mathrm{E} \times \mathrm{H}$ ).

\section{ERD}

Figure 4 presents the time course of ERD/ERS at selected channels; Figure 5 presents topographic maps of the pre-KR ERD for all conditions. The premovement ERD follows the pattern of results usually reported (e.g., Pfurtscheller \& Berghold, 1989). 
a)

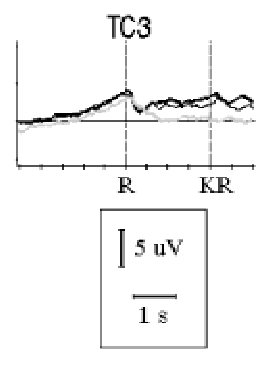

SPN time courses
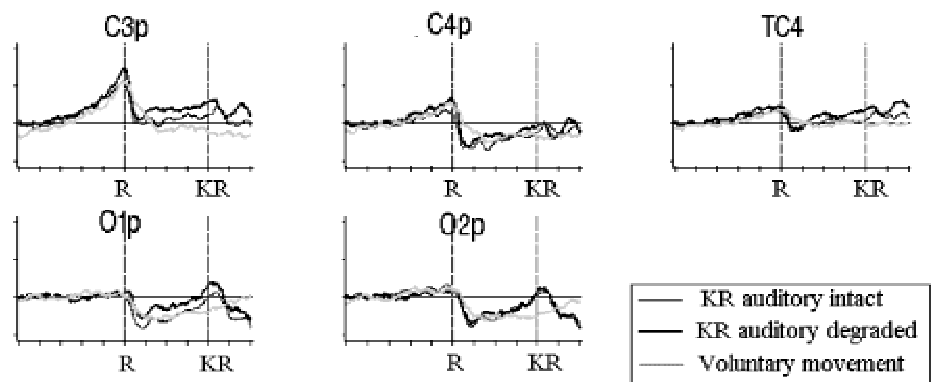

b)
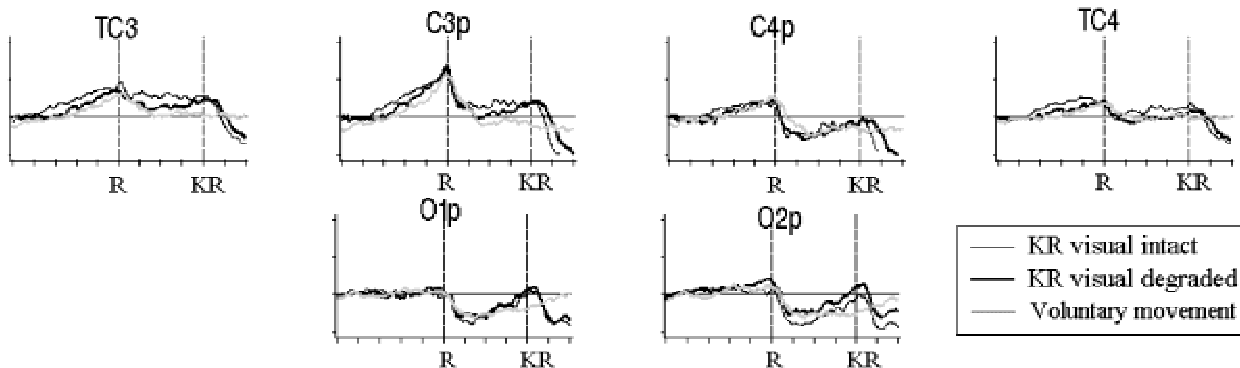

KR visual intact

- KR visual degraded

_ Voluntary movement

Figure 2. Time courses of the grand average $(N=12)$ slow potentials at selected channels. Negativity is plotted upwards. Data are from right-hand movements. (a) Comparison between both auditory time-estimation conditions and the voluntary-movement condition. (b) Comparison between both visual time-estimation conditions and the voluntary-movement condition. Note (1) that the presentation of a KR stimulus results in a stimulus-preceding negativity (SPN) that is not present in the voluntary-movement condition; (2) that preceding auditory KR stimuli, the SPN amplitude is larger when stimuli are degraded, and (3) that this effect is not present for KR stimuli in the visual modality.

Starting from approximately $1,250 \mathrm{~ms}$ prior to movement onset, an ERD can be observed over the contralateral motor cortex. This contralateral ERD shifts to a bilaterally symmetrical ERD just prior to movement onset. Prior to the presentation of the KR stimulus, at central channels a massive postmovement ERS can be observed over the contralateral motor cortex, as previously described by, for example, Pfurtscheller (1992). Prior to visual KR stimuli, an additional occipital ERD is present, whereas prior to auditory KR stimuli no appreciable ERD is observed.

Alpha power in the reference interval. The ANOVAs on the level of absolute alpha power in the reference interval revealed that there were no differences in baseline power between conditions. A main effect of electrode pair $(8-10 \mathrm{~Hz}$ band: $F(3,33)=7.33, p=$ $.0203, \epsilon=.33 ; 10-12 \mathrm{~Hz}$ band: $F(3,33)=3.81, p=.0769, \epsilon=$ .33) indicated that the power was largest at occipital leads. Furthermore, in both frequency bands, a main effect of hemisphere $(8-10 \mathrm{~Hz}: F(1,11)=11.00, p=.0069 ; 10-12 \mathrm{~Hz}: F(1,11)=5.43$, $p=.0399)$ indicated that the power was larger in the right hemisphere $\left(8-10 \mathrm{~Hz}: 327 \pm 37\left(\mathrm{~A} / \mathrm{m}^{2}\right)^{2} ; 10-12 \mathrm{~Hz}: 487 \pm 71\right.$ $\left.\left(\mathrm{A} / \mathrm{m}^{2}\right)^{2}\right)$ than in the left hemisphere $\left(8-10 \mathrm{~Hz}: 161 \pm 27\left(\mathrm{~A} / \mathrm{m}^{2}\right)^{2}\right.$; 10-12 Hz: $\left.247 \pm 486\left(\mathrm{~A} / \mathrm{m}^{2}\right)^{2}\right)$. The absence of effects involving the factor Condition indicates that differences in ERD between conditions cannot be attributed to differences in the power in the reference interval.

Movement-related ERD. The results of the ANOVA on the movement-related ERD are summarized in Table 3. The movement- related ERD is globally characterized by three effects, which are expressed in the interaction of response side with time interval and hemisphere $(\mathrm{R} \times \mathrm{I} \times \mathrm{H})$ : First, at the early premovement time interval, there is a contralaterally dominant ERD (simple effect of $\mathrm{R} \times \mathrm{H}$ at this time interval: $F(1,11)=5.15, p=.0444)$. Second, at the late premovement time interval, there is a bilaterally symmetrical ERD, which somewhat surprisingly displays a trend towards a larger ERD on the ipsilateral side (simple effect of $\mathrm{R} \times \mathrm{H}$ at this time interval: $F(1,11)=3.71, p=.0802$ ). Third, at the postmovement time interval (which corresponds to the pre-KR interval in the time estimation conditions) there is a contralaterally dominant ERS (simple effect of $\mathrm{R} \times \mathrm{H}$ at this time interval: $F(1,11)=15.52, p=.0023)$. However, the contralateral dominance of the postmovement ERS is significant only following right-hand movements (simple effect of hemisphere in right-hand movement conditions at this time interval: $F(1,11)=14.04, p=$ .0032), whereas following left-hand movements, the postmovement ERS is bilaterally symmetrical. This can be seen in Figure 5. Furthermore, the magnitude of the postmovement ERS following right-hand responses is such that it also produces the main effects of Time Interval and Hemisphere, and the interaction of Time Interval with Hemisphere.

However, the effects contained in the $\mathrm{R} \times \mathrm{I} \times \mathrm{H}$ interaction only hold for the time-estimation conditions, as indicated by the fourth-order interaction Condition $\times$ Response Side $\times$ Time Interval $\times$ Hemisphere: the simple effect of $\mathrm{R} \times \mathrm{I} \times \mathrm{H}$ is significant in all four time-estimation conditions (all $p$ values $<.05$ ) but not in the voluntary-movement condition. This can be attributed to the 

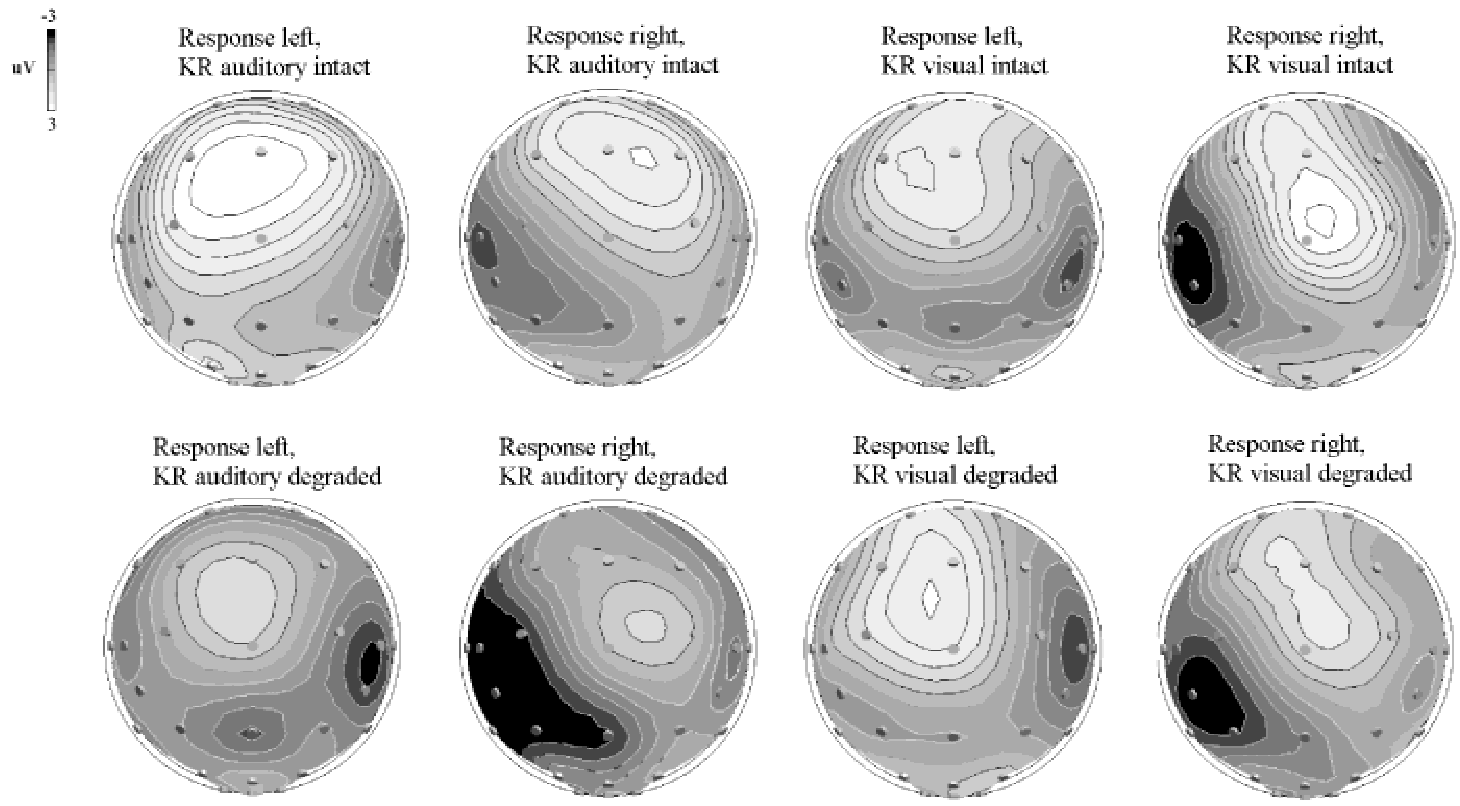

Figure 3. Topographic maps of the stimulus-preceding negativity (SPN), averaged over the last 100 -ms interval preceding stimulus presentation, in all time-estimation conditions. Positivity is depicted as light gray surrounded by black isopotential lines; negativity is depicted as dark gray surrounded by white isopotential lines. Spacing of isopotential lines is $0.5 \mu \mathrm{V}$. Scaling as indicated in the figure. Note (1) that only in the auditory modality, the amplitude of the SPN increases with stimulus degradation, and (2) that the scalp distribution of the SPN is independent of stimulus modality.

fact that in the voluntary-movement conditions, the postmovement ERS is much smaller than in the time-estimation conditions.

The differences in postmovement ERS between the voluntarymovement conditions and the time-estimation conditions indicates that we cannot subtract the ERD in the voluntary-movement task from the ERD in the time-estimation task to eliminate the movementrelated ERD from the pre-KR ERD. However, if we can demonstrate that the postmovement ERS at central channels is similar for all time-estimation conditions, we can still validly attribute differences in ERD at other channels to prestimulus processes. This was verified by an additional ANOVA on the postmovement ERS in the time-estimation conditions at central channels in the pre-KR interval, with variables frequency band $(8-10 \mathrm{~Hz}, 10-12 \mathrm{~Hz})$, con- dition (auditory intact, auditory degraded, visual intact, visual degraded), response side (left hand, right hand) and hemisphere (left, right). This ANOVA revealed no effects involving the variable condition (all $p$ values $>.18$ ). Significant effects were response side, $F(1,11)=5.86, p=.0340$, indicating that the postmovement ERS was larger following right-hand responses than following left-hand responses $(91 \pm 13 \%$ and $60 \pm 9 \%$ power increase, respectively) and hemisphere, $F(1,11)=4.78, p=.0490$, indicating that ERS was larger in the left than in the right hemisphere $(133 \pm 14 \%$ and $38 \pm 6 \%$ power increase, respectively). An interaction of response side with hemisphere, $F(1,11)=15.55, p=$ .0023 , further indicates that these effects are due to the fact that following right-hand responses, the ERS is present exclusively on
Table 1. Significant Effects of the ANOVA on the Stimulus-Preceding Negativity

\begin{tabular}{lrrrrr}
\hline \hline Effect & $F$ & $d f$ & $p$ & $\epsilon$ \\
\hline KR type (T) & 6.83 & 1,11 & .0239 & \\
Electrode pair (E) & 7.20 & 3,33 & .0027 & .74 \\
KR Modality (M) X KR Type & 4.74 & 1,11 & .0521 & \\
Response Side (R) X Hemisphere (H) & 44.60 & 1,11 & $<.0001$ & \\
Modality X Type X Hemisphere & 5.32 & 1,11 & .0415 & \\
Response Side X Electrode Pair $\times$ Hemisphere & 18.32 & 3,33 & .0001 & .56 \\
& & & &
\end{tabular}

Note: Reported $p$ values are corrected with the Greenhouse-Geisser epsilon $(\epsilon)$
Table 2. SPN Amplitudes in the Different Conditions, Averaged Over Response Sides and Hemispheres ${ }^{\text {a }}$

\begin{tabular}{|c|c|c|c|c|}
\hline \multicolumn{5}{|c|}{ SPN amplitudes } \\
\hline KR Type & \multicolumn{2}{|c|}{ Intact } & \multicolumn{2}{|c|}{ Degraded } \\
\hline KR Modality & Auditory & Visual & Auditory & Visual \\
\hline Temporal Electrodes & $-1.0(0.3)$ & $-1.7(0.4)$ & $-3.7(0.3)$ & $-1.2(0.4)$ \\
\hline Occipital Electrodes & $0.7(0.4)$ & $0.3(0.4)$ & $-0.7(0.4)$ & $0.0(0.4)$ \\
\hline
\end{tabular}

${ }^{a}$ Note that in the auditory KR conditions only, SPN amplitudes increase (that is, become more negative) with stimulus degradation. 
a)

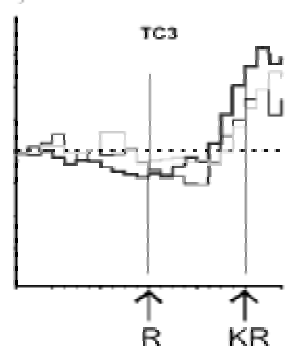

ERD time courses
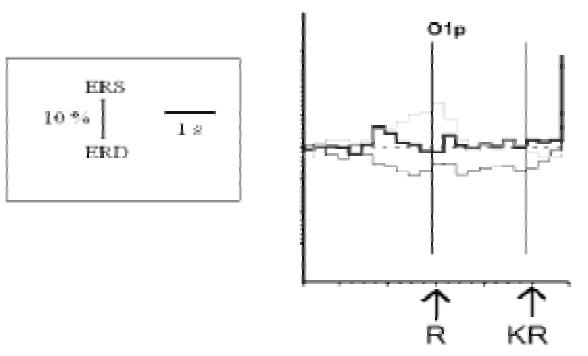
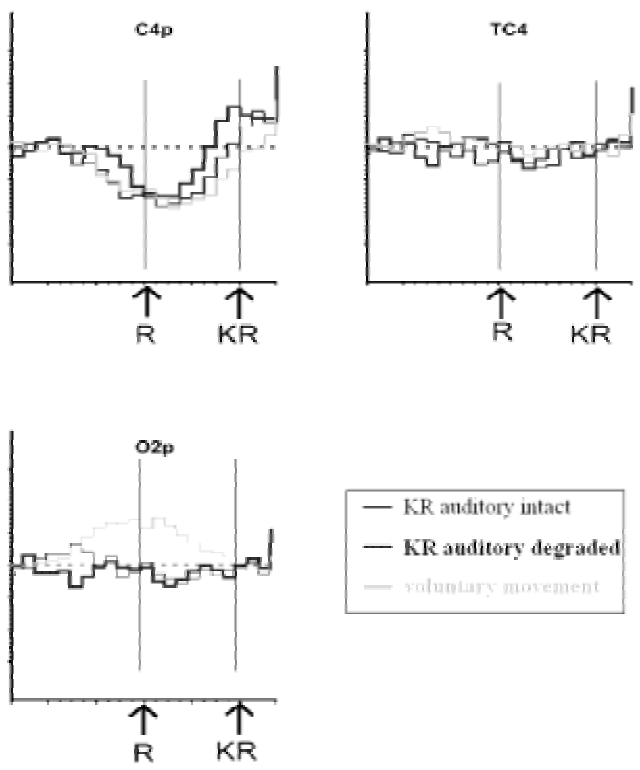

b)
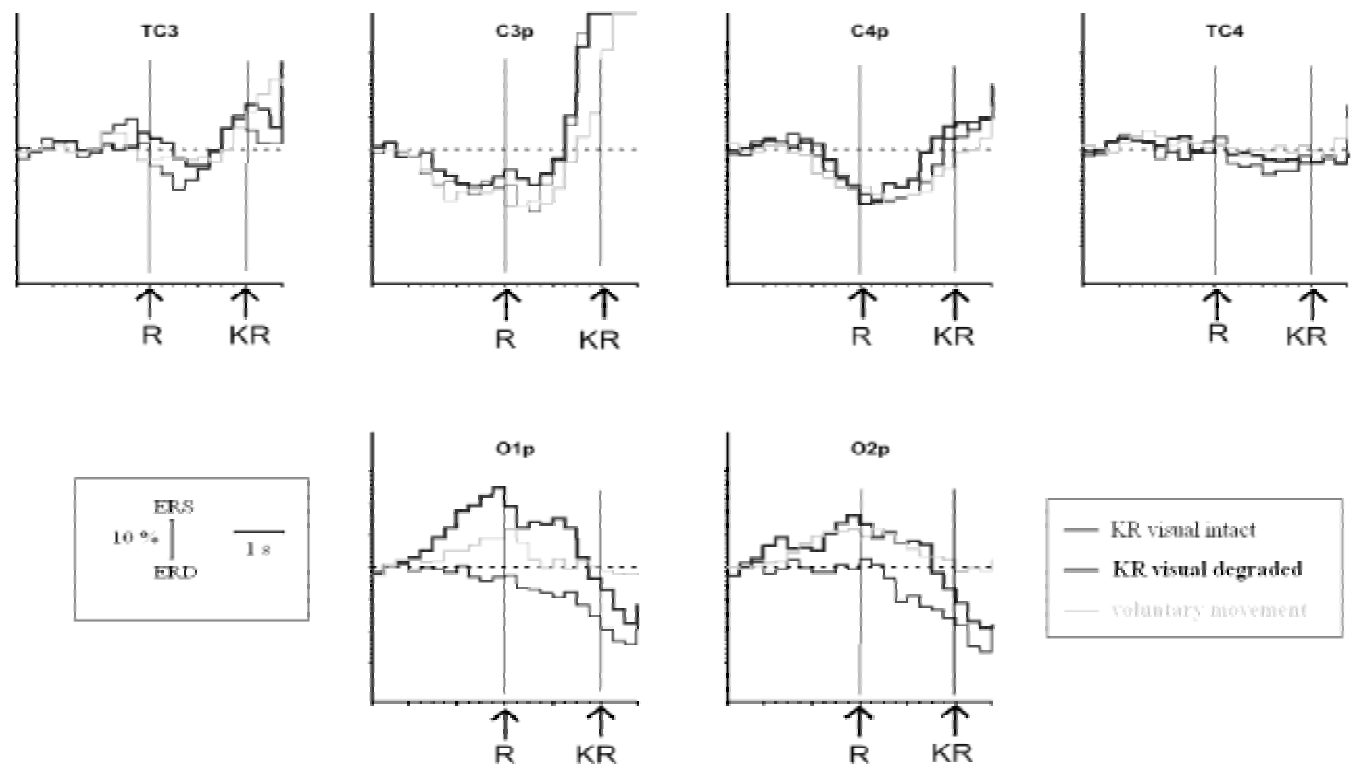

- KR visual degraded

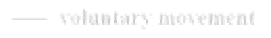

Figure 4. Time courses of the grand average $(N=12)$ power changes (ERD/ERS) in the $10-12 \mathrm{~Hz}$ frequency band at selected channels. Data are from right-hand movements. Positive values indicate power increase (ERS); negative values indicate power decrease (ERD). (a) Comparison between both auditory time-estimation conditions and the voluntary-movement condition. (b) Comparison between both visual time-estimation conditions and the voluntary-movement condition. Note that, because ERD is computed in 250-ms intervals, the time courses are represented as a step function.

the contralateral side (simple effect of hemisphere at right hand conditions: $F(1,11)=12.74, p=.0044$, whereas following lefthand responses the ERS is bilaterally symmetrical (simple effect of hemisphere at left hand conditions: $F(1,11)=.00, p=.9798)$; see also Figure 5.

Prestimulus ERD. Table 4 presents the results of the $t$ tests on the prestimulus ERD. As Table 4 reveals, prior to the presentation of visual KR stimuli, there is a significant ERD at occipital elec- trode positions, but not at temporal electrodes. Preceding auditory KR stimuli, there is no significant ERD; in the 10-12 Hz band, there is even a significant ERS at temporal electrode positions preceding auditory degraded KR stimuli.

The differences in movement-related ERD between conditions prohibit a subtraction of the ERD in the voluntary-movement conditions from the ERD in the time-estimation conditions. Therefore the ANOVA on the prestimulus ERD was performed on the nonsubtracted data. As a consequence, the results of the ANOVA 


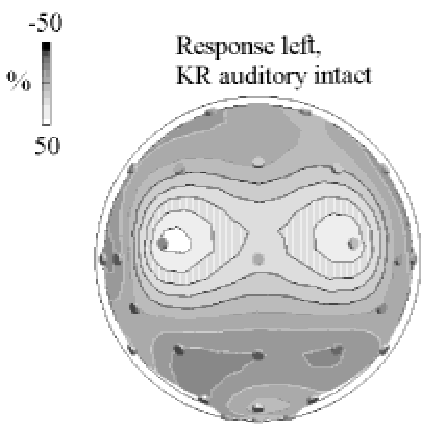

\section{Scalp topography of the prestimulus ERD}
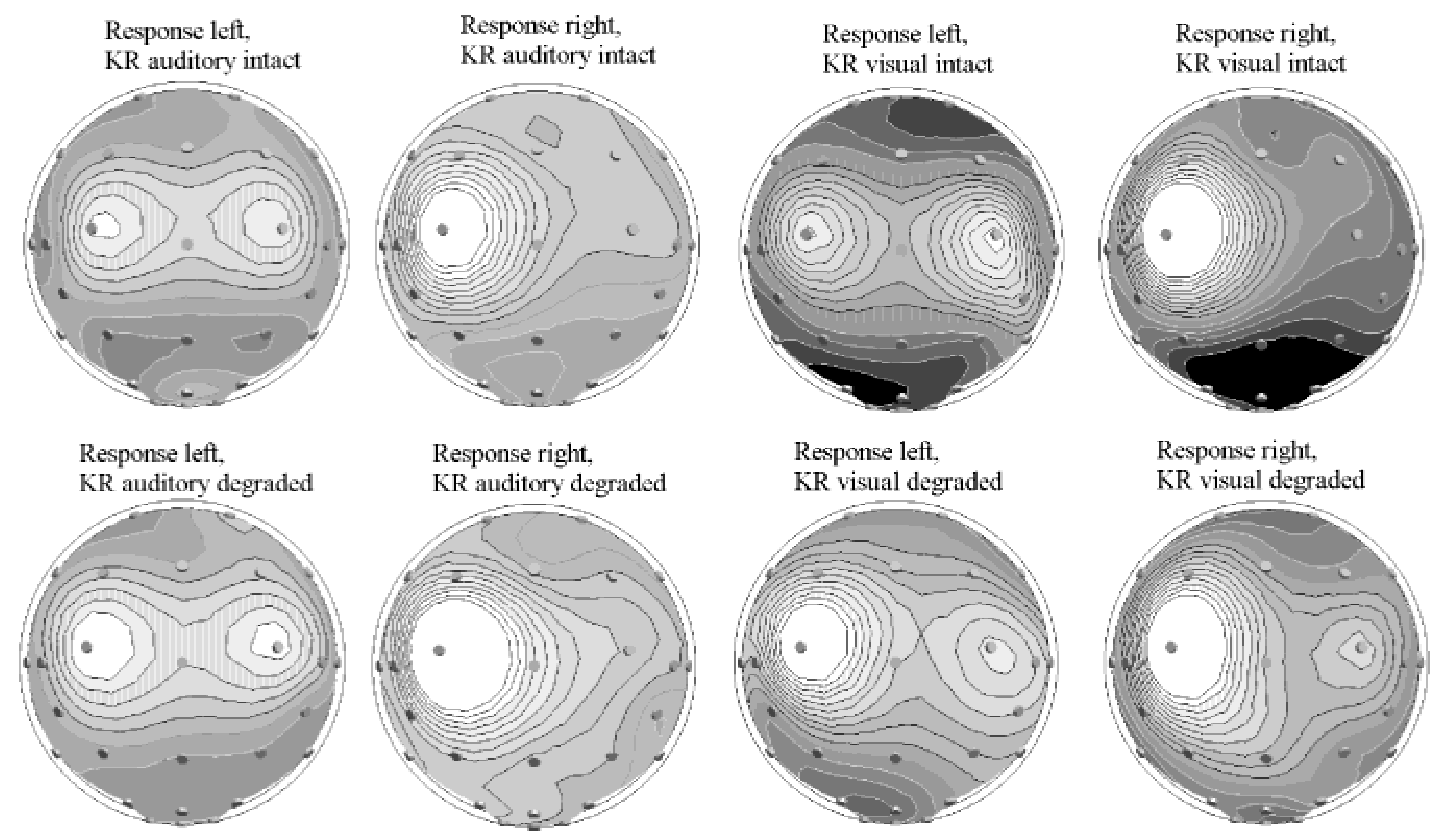

Figure 5. Topographic maps of the grand average $(N=12)$ ERD in the last 250-ms interval preceding the presentation of the KR stimulus. Power increase (ERS) is depicted as light gray surrounded by black isopotential lines; power decrease (ERD) is depicted as dark gray surrounded by white isopotential lines. Spacing of isopercentage lines is $5 \%$. Scaling as indicated in the figure. Note (1) the strong postmovement ERS at central electrodes, (2) that the ERD preceding the presentation of the KR stimuli (right-hand column) is dependent upon stimulus modality: preceding auditory stimuli, no ERD is present, whereas preceding visual stimuli, an occipital ERD is present, and (3) that the latter is larger preceding intact than preceding degraded stimuli.

on the prestimulus ERD (summarized in Table 5) show a number of effects that can be attributed to the postmovement ERS rather than to prestimulus processes: A main effect of electrode pair reveals that there is an ERD at occipital electrode positions (17 \pm $1.7 \%$ power decrease) whereas at temporal electrode positions there is an ERS ( $8 \pm 2.4 \%$ power increase). Visual inspection of Figures 4 and 5 supports the interpretation of the latter as a postmovement ERS that extends from central to temporal electrode positions. Furthermore, the interaction of response side with hemisphere $(\mathrm{R} \times \mathrm{H})$ reveals that in right-hand movement condi-

Table 3. Significant Effects of the ANOVA on the Movement-Related ERD ${ }^{\text {a }}$

\begin{tabular}{lrccc}
\hline \hline Effect & \multicolumn{1}{c}{$F$} & $d f$ & $p$ & $\begin{array}{c}\mathrm{GG} \\
\text { Epsilon }\end{array}$ \\
\hline Time Interval (I) & 16.49 & 2,22 & .0014 & .53725 \\
Hemisphere (H) & 5.71 & 1,11 & .0359 & \\
Response Side (R) $\times$ Hemisphere & 12.65 & 1,11 & .0045 & \\
$\mathrm{I} \times \mathrm{H}$ & 4.91 & 2,22 & .0474 & .51355 \\
$\mathrm{R} \times \mathrm{I} \times \mathrm{H}$ & 16.42 & 2,22 & .0014 & .54220 \\
Condition $\times \mathrm{R} \times \mathrm{I} \times \mathrm{H}$ & 3.51 & 8,88 & .0328 & .32520 \\
& & & & \\
\hline \hline
\end{tabular}

${ }^{\text {a}}$ Reported $p$ values are corrected with the Greenhouse-Geisser epsilon $(\epsilon)$. tions, there is a significant, $F(1,11)=8.15, p=.0157$, difference between the right $(9 \pm 2.5 \%$ power decrease) and left $(4 \pm 3.7 \%$ power increase) hemispheres, which is not present in left-hand movement conditions. The interaction of response side with electrode pair and hemisphere $(\mathrm{R} \times \mathrm{E} \times \mathrm{H})$ indicates that this effect is restricted to temporal electrode positions. These effects $(\mathrm{R} \times \mathrm{H}$ and $\mathrm{R} \times \mathrm{E} \times \mathrm{H}$ ) are probably produced by the fact that in right-hand movement conditions, there is a strong contralaterally dominant ERS, whereas in left-hand movement conditions the postmovement ERS is bilaterally symmetrical. Finally, the interaction of frequency band with KR modality and response side indicates that in the $8-10 \mathrm{~Hz}$ band, following right-hand responses, there is a significant difference, $F(1,11)=7.29, p=.0207$, between the ERD preceding auditory $(13 \pm 5 \%$ power increase) and visual (13 $\pm 4 \%$ power decrease) KR stimuli, which is not present following left-hand movements. Although this does not interact with electrode pair, it is probable that this effect is caused by the differences in postmovement ERS between right- and lefthand responses.

The remaining two effects are not easily explained in terms of postmovement processes, and probably truly reflect prestimulus processes. First, a trend towards a main effect of modality indicated that the ERD tended to be larger preceding visual $(10 \pm 2 \%$ power decrease) than preceding auditory ( $2 \pm 2 \%$ power increase) KR stimuli. Second, contrary to expectation, the ERD was smaller preceding degraded KR stimuli $(1 \pm 2 \%$ power increase $)$ than preceding intact KR stimuli ( $9 \pm 2 \%$ power decrease), as indicated 
Table 4. Results of the t tests on the prestimulus ERD, averaged over response sides and hemispheres.

\begin{tabular}{|c|c|c|c|c|c|c|c|c|}
\hline \multirow{3}{*}{$\begin{array}{c}\text { Freq. Band } \\
\text { KR Type } \\
\text { KR Modality }\end{array}$} & \multicolumn{4}{|c|}{$8-10 \mathrm{~Hz}$} & \multicolumn{4}{|c|}{$10-12 \mathrm{~Hz}$} \\
\hline & \multicolumn{2}{|c|}{ Intact } & \multicolumn{2}{|c|}{ Degraded } & \multicolumn{2}{|c|}{ Intact } & \multicolumn{2}{|c|}{ Degraded } \\
\hline & Auditory & Visual & Auditory & Visual & Auditory & Visual & Auditory & Visual \\
\hline Temporal Electrodes & $10(8)$ & $-1(8)$ & $11(6)$ & $11(7)$ & $8(6)$ & $2(6)$ & $20(7) *$ & $5(5)$ \\
\hline Occipital Electrodes & $-8(5)$ & $-33(5) * *$ & $0(5)$ & $-18(5) *$ & $-4(4)$ & $-31(3) * * *$ & $-8(5)$ & $-21(5) *$ \\
\hline
\end{tabular}

Note: In each cell the average (standard error) percentage power change is given; negative values indicate a power decrease (ERD), positive values indicate a power increase (ERS). Note (1) that the prestimulus ERD is only significant at occipital electrodes preceding visual stimuli, both for the intact stimuli and for the degraded stimuli, and (2) that the ERD is larger preceding the intact than preceding the degraded stimuli. This difference is significant as revealed by the ANOVA (see Table 5). $* p<.05 ; * * p<.01 ; * * * p<.001$.

by a main effect of KR Type. Both effects can be seen in Figures 4 and 5 .

\section{Discussion}

We investigated the stimulus-preceding negativity and the eventrelated changes of the power in the alpha frequency band (ERD/ ERS) of the EEG related to the anticipation of auditory and visual stimuli, in a paradigm in which subjects performed a timeestimation task and were informed about the quality of their time estimation by a KR stimulus. The perceptual anticipation was manipulated by presenting the stimuli either in intact or in degraded form. The modality of the KR stimulus did not influence the scalp topography of the SPN. However, the SPN was larger preceding degraded auditory KR stimuli than in the other three conditions. This coincided with a reduced effectiveness of the KR stimulus in guiding the behavior on the next trial in the former condition. In contrast, we found clear differences between modalities in the prestimulus ERD: preceding auditory KR stimuli no ERD was present, whereas the visual KR was preceded by an ERD over the occipital areas (cf. Table 4; Bastiaansen, Böcker, et al., 1999). Contrary to expectation, the occipital ERD was smaller preceding degraded visual KR stimuli than when intact stimuli were presented.

In the following paragraphs, we will first discuss the slow potentials and the ERD related to the anticipation of the KR stimulus, because this is the main focus of the present article. Next, we will discuss in more detail the results of the slow potentials and

Table 5. Significant Effects of the ANOVA on the Prestimulus ERD ${ }^{\text {a }}$

\begin{tabular}{lrcc}
\hline \hline Effect & \multicolumn{1}{c}{$F$} & $d f$ & $p$ \\
\hline KR Modality $(\mathrm{M})$ & 4.37 & 1,11 & .0607 \\
KR Type & 8.34 & 1,11 & .0148 \\
Electrode pair $(\mathrm{E})$ & 13.76 & 1,11 & .0034 \\
Response Side $(\mathrm{R}) \times$ Hemisphere $(\mathrm{H})$ & 10.96 & 1,11 & .0069 \\
$\mathrm{R} \times \mathrm{E} \times \mathrm{H}$ & 7.19 & 1,11 & .0214 \\
Frequency Band $\times \mathrm{M} \times \mathrm{R}$ & 14.20 & 1,11 & .0031 \\
\hline
\end{tabular}

${ }^{a}$ Note that the main effect of KR Modality is only marginally significant. the ERD related to movement execution, because the results are striking in some respects.

\section{Prestimulus processes}

The SPN was larger preceding auditory degraded stimuli than preceding the other three types of KR stimuli (auditory intact, visual degraded, and visual intact). In this respect, it is interesting to note that the effectiveness of the degraded auditory KR stimuli was lower than for the other KR stimuli in guiding the behavior on the subsequent trial. This indicates that these stimuli were more difficult to recognize, and, as a result, the task was more difficult to perform. A possible interpretation of these findings would be that subjects counteracted the reduced effectiveness of the degraded auditory KR stimuli by an increase in motivation or effort to accurately perform the task, which produced an enhanced SPN amplitude. Although independently our data provide only weak evidence for this notion, such an interpretation is well in line with previous studies (Chwilla \& Brunia, 1991b, 1992), which have shown that SPN amplitudes increase with increasing task difficulty. Thus, our data support the interpretation of the pre-KR SPN as reflecting anticipation of the affective-motivational aspects of knowledge of results rather than the perceptual anticipatory attention that is described by our neurophysiological model (Brunia, 1999). Furthermore, it should be noted that task difficulty was not intentionally manipulated: in the behavioral pilot experiment, in which the level of stimulus degradation was determined through a classification task, stimulus degradation influenced reaction times but not percent correct classifications. The increase in task difficulty due to degradation of the auditory stimuli in the actual experiment might be tentatively explained by assuming that in the classification task it was easier to discriminate between the three auditory degraded KR stimuli because they were presented at a higher rate (once every $2 \mathrm{~s}$ ) than in the actual time-estimation task, thereby allowing for a better comparison between each new stimulus and the preceding one(s). However, regardless of what may have caused the unexpected increase in task difficulty, the relevant finding for the present purpose is that it affected the SPN amplitude, whereas a mere increase in perceptual difficulty, as in the visual modality, did not lead to an increase in SPN amplitude.

In general terms, the results of the prestimulus ERD, that is, an occipital ERD prior to visual KR stimuli and an absence of ERD prior to auditory stimuli, are a replication of the results of previous experiments (Bastiaansen, Böcker, et al., 1999; Bastiaansen, Brunia, et al., 1999; Bastiaansen et al., in press). We have explained 
the absence of a bilateral temporal ERD in the EEG in terms of a differential sensitivity of EEG and MEG for rhythmic activity originating from the auditory cortex (cf. Bastiaansen, Böcker, et al., 1999; Lehtelä et al., 1997; Tiihonen et al., 1991), which has been partly confirmed by the results of a subsequent study combining EEG and MEG (Bastiaansen et al., 2001). In addition, we have tentatively interpreted the occipital ERD prior to visual stimuli as reflecting anticipatory attention (Bastiaansen, Böcker, et al., 1999; Bastiaansen, Brunia, et al., 1999). As we pointed out in the Introduction, the latter interpretation would gain strength if the pre-KR ERD were to be influenced by stimulus parameters. In the behavioral pilot experiment, we have established that it took subjects more time to classify the degraded stimuli than the intact stimuli, which strongly suggests that the former were more difficult to perceive. We have reasoned that in such a situation anticipatory attention would be more prominent than in a situation in which stimuli are easily perceived. Therefore, if the prestimulus ERD is a correlate of a perceptually based anticipatory attention process, we would expect it to be larger preceding degraded stimuli than preceding intact stimuli. Although the finding of the present experiment that stimulus degradation results in a smaller ERD (although still significantly different from zero) does indicate a sensitivity of the anticipatory ERD to stimulus parameters, the direction of the effect is opposite to what we expected. It is unclear at present how to interpret this effect.

Finally, it should be noted that with the exception of an intricate third-order interaction including response side, which probably reflects postmovement processes, we did not find any differences between the upper and lower alpha bands in any of the analyses on prestimulus ERD. This argues against the hypothesis by Klimesch and coworkers (e.g., Klimesch, 1999) that the lower alpha band is more related to attention and/or anticipation (expectancy in their terminology) than the upper alpha band. However, this negative finding may well be explained by the fact that in the present study, fixed frequency bands were used instead of individually adjusted frequency bands. Indeed, Klimesch (1999) has shown that using fixed frequency bands may, in some cases, blur true differences in upper and lower alpha bands, due to the fact that individuals differ in their individual alpha peak frequencies. Using frequency bands adjusted on the basis of the individual alpha peak may help to better reveal possible differences in the prestimulus ERD in the upper and lower alpha bands in future research.

\section{Movement-Related Processes}

The RP that was observed prior to movement execution did not show any differences between conditions. However, the analyses of the movement-related ERD indicated that although the premovement ERD was similar for all conditions, there was a marked difference in the magnitude of the ERS at central positions following the movement between the time-estimation task and the voluntary-movement task: in the former task the ERS was much larger than in the latter. A point of concern, therefore, is whether this effect is a reflection of postmovement processes, or whether it is somehow related to the anticipation of the KR stimulus. It seems unlikely for several reasons that such a synchronization at central electrode positions can be attributed to the anticipation of the KR stimulus. First, it is a well-established phenomenon that (both voluntary and externally paced) movements are followed by ERS in the alpha and beta bands (e.g., Pfurtscheller, Zalaudek, \& Neuper, 1998; Stancak \& Pfurtscheller, 1995, for beta ERS, and, e.g., Bastiaansen, Böcker, et al., 1999; Leocani, Toro, Manganotti, Zhuang, \& Hallet, 1997; Stancak \& Pfurtscheller, 1996; Toro et al., 1994, for alpha ERS). Second, there is no qualitative, but only a quantitative difference between the ERS in the time estimation tasks and the voluntary movement task in the present study: The ERS observed after the movement in the present study has a central maximum and a contralateral dominance that is most pronounced following right-hand movements in all three conditions (i.e., including the voluntary movement condition).

Thus, we feel there are sufficient arguments to interpret the ERS observed after the movement in the time estimation tasks in terms of postmovement processes. The question is, then, why was this postmovement ERS larger in the time-estimation conditions than in the voluntary-movement condition? Because the alpha ERS has been shown to vary with movement parameters (e.g., Cassim et al., 2000) just as the beta ERS (although the latter phenomenon has been studied more extensively, e.g., Pfurtscheller, Zalaudek, \& Neuper, 1998; Salmelin, Hämäläinen, Kajola, \& Hari, 1995; Stancak \& Pfurtscheller, 1995; Stancak, Riml, \& Pfurtscheller, 1997; Toro et al., 1994), it seems reasonable to attribute this effect to differences in movement parameters between the voluntarymovement and the time-estimation conditions. These differences in movement parameters may have been induced by the high constraints on the timing of the movement in the latter conditions, which were not present in the former. Unfortunately we cannot demonstrate differences in movement parameters, because we did not measure EMG. However, the lack of qualitative differences between the voluntary-movement and the time-estimation conditions does support this interpretation. Finally, it must be noted that our findings in this respect contrast with the results of a previous study, where we did not find any differences in movement-related ERD/ERS between both tasks (Bastiaansen, Böcker, et al., 1999). Further research would be needed to clarify this issue.

In conclusion, in the main, our results support a clear dissociation between the SPN and the anticipatory ERD. Our present data are in agreement with the notion that the pre-KR SPN is mainly a reflection of the affective-motivational anticipation of a stimulus that evaluates past performance, in that it is larger in the more difficult condition. In addition, although the exact functional significance of the anticipatory ERD needs to be investigated by further delineating its antecedent conditions, the results globally support the idea that the anticipatory ERD is related to perceptual anticipatory attention because it was influenced by stimulus degradation.

\section{REFERENCES}

Bastiaansen, M. C. M., Böcker, K. B. E., Cluitmans, P. J. M., \& Brunia, C. H. M. (1999). Event-related desynchronization related to the anticipation of a stimulus providing knowledge of results. Clinical Neurophysiology, 110, 250-260.

Bastiaansen, M. C. M., Böcker, K. B.E., Brunia, C. H. M., de Munck, J. C., \& Speckreijse, H. (2001). Event-related desynchronization during anticipatory attention for an upcoming stimulus: A comparative EEG/ MEG study. Clinical Neurophysiology, 112, 393-403.
Bastiaansen, M. C. M., Brunia, C. H. M., \& Böcker, K. B. E. (1999). ERD as an index of anticipatory behavior. In: G. Pfurtscheller, F. Lopez da Silva (Eds.) Event-related desynchronization. Handbook of Electroencephalography and Clinical Neurophysiology, revised series (Vol. 6, pp. 203-217). Amsterdam: Elsevier.

Birbaumer, N., Elbert, T., Canavan, A., \& Rockstroh, B. (1990). Slow potentials of the cerebral cortex and behavior. Physiological Review, $70,1-41$. 
Böcker, K. B. E., Brunia, C. H. M., \& Van den Berg-Lenssen, M. M. C. (1994). A spatiotemporal dipole model of the stimulus-preceding negativity (SPN) prior to feedback stimuli. Brain Topography, 7, 71-88.

Böcker, K. B. E. \& Van Boxtel, G. J. M. (1997). Stimulus-preceding negativity: A class of anticipatory slow potentials. In: G. J. M. van Boxtel \& K. B. E. Böcker (Eds.), Brain and behavior. Past, present and future (pp. 105-116). Tilburg: Tilburg University Press.

Brunia, C. H. M. (1993). Waiting in readiness: Gating in attention and motor preparation. Psychophysiology, 30, 327-339.

Brunia, C. H. M. (1999). Neural aspects of anticipatory behavior. Acta Psychologica, 101, 213-242.

Brunia, C. H. M., \& Damen, E. J. P. (1988). Distribution of slow potentials related to motor preparation and stimulus anticipation in a time estimation task. Electroencephalography and Clinical Neurophysiology, 69, 234-243.

Brunia, C. H. M., De Jong, B.M., Van Den Berg-Lenssen, M. M. C. and Paans, A. M. J. (2000). Visual feedback about time estimation is related to a right-hemisphere activation measured by PET. Experimental Brain Research, 130, 328-337.

Brunia, C. H. M., \& Van Boxtel, G. J. M. (in press). Wait and see. International Journal of Psychophysiology.

Cassim, F., Szurhaj, W., Sediri, H., Devos, D., Bourriez, J.-L., Poirot, I., Derambure, P., Defebvre, L., \& Guieu, J.-D. (2000). Brief and sustained movements: Differences in event-related (de)synchronization (ERD/ ERS) patterns. Clinical Neurophysiology, 111, 2032-2039.

Chwilla, D. J., \& Brunia, C. H. M. (1991a). Event-related potentials to different feedback stimuli. Psychophysiology, 28, 123-132.

Chwilla, D. J., \& Brunia, C. H. M. (1991b). Event-related potential correlates of non-motor anticipation. Biological Psychology, 32, 125-141.

Chwilla, D. J. \& Brunia, C. H. M. (1992). Effects of emotion on eventrelated potentials in an arithmetic task. Journal of Psychophysiology, 6 , 321-332.

Damen, E. J. P., \& Brunia, C. H. M. (1987). Changes in heart rate and slow brain potentials related to motor preparation and stimulus anticipation in a time estimation task. Psychophysiology, 24, 700-713.

Damen, E. J. P., Brunia, C. H. M. (1994). Is a stimulus conveying taskrelevant information a sufficient condition to elicit a Stimulus-Preceding Negativity? Psychophysiology, 31, 129-139.

Gaillard, A. W. K., \& Van Beijsterveld, C. E. M. (1991). Slow brain potentials elicited by a cue signal. Journal of Psychophysiology, 5, 337-347.

Grünewald, G., Grünewald-Zuberbier, E., Hömberg, V., \& Schuhmacher, H. (1984). Hemispheric asymmetry of feedback-related slow negative potential shifts in a positioning movement task. In: R. Karrer \& P. Tueting (Eds.), Brain and information: Event-related potentials. (pp. 470-476). New York: New York Academy of Sciences.

Hari, R., Salmelin, R., Mäkelä, J. P., Salenius, S., Helle, M. (1997). Magnetoencephalographic cortical rhythms. International Journal of Psychophysiology, 26, 51-62.

Hjorth, B. (1975). An on-line transformation of EEG scalp potentials into orthogonal source derivations. Electroencephalography and Clinical Neurophysiology, 39, 526-530.

Klimesch, W. (1999). EEG alpha and theta oscillations reflect cognitive and memory performance: A review and analysis. Brain Research Reviews, 29, 169-195.

Klimesch, W., Doppelmayr, M., Russegger, H., Pachinger, Th., \& Schweiger, J. (1998). Induced alpha band power changes in the human EEG and attention. Neuroscience Letters, 244, 73-76.

Klimesch, W., Pfurtscheller, G., \& Schimke, H. (1992) Pre- and poststimulus processes in category judgment tasks as measured by event-related desynchronization (ERD). Journal of Psychophysiology, 6, 186-203.

Kornhuber, K. K., Deecke, L. (1965). Hirnpotentialänderungen bei Willkürbewegungen und passiven Bewegungen des Menschen: Bereitschaftspotential und reafferente Potentiale. Pflügers Archiv, 284, 1-17.

Kotani, Y., \& Aihara, Y. (1999). The effect of stimulus discriminability on stimulus-preceding negativities prior to instructive and feedback stimuli. Biological Psychology, 50, 1-18.

Kotani, Y., Hiraku, S., \& Aihara, Y. (1999). Effects of affective-motivational valence on the stimulus-preceding negativity prior to feedback stimuli. Psychophysiology, 36, S70.

Lehtelä, L., Salmelin, R., \& Hari, R. (1997). Evidence for reactive magnetic $10-\mathrm{Hz}$ rhythm in the human auditory cortex. Neuroscience Letters, 222, 111-114.
Leocani, L., Toro, C., Manganotti, P., Zhuang, P., \& Hallet, M. (1997). Event-related coherence and event-related desynchronization/synchronization in the $10 \mathrm{~Hz}$ and $20 \mathrm{~Hz}$ EEG during self-paced movements. Electroencephalography and Clinical Neurophysiology, 104, 199-206.

Lopes da Silva, F.H. (1991). Neural mechanisms underlying brain waves: From neural membranes to networks. Electroencephalography and Clinical Neurophysiology, 79, 81-93.

Lopes da Silva, F. H., \& Pfurtscheller, G. (1999). Brain oscillations and event-related dynamics. In: G. Pfurtscheller \& F. H. Lopes da Silva (Eds.), Event-related desynchronization. Handbook of Electroencephalography and Clinical Neurophysiology, revised series, (Vol. 6, pp. 313). Amsterdam: Elsevier.

Mesulam, M. M., \& Mufson, J.E. (1985). The insulae of Reil in man and monkey. Architectonics, connectivity and function. In: A. Peters \& E. G. Jones (Eds.), Cerebral cortex. Vol. 4., Association and auditory cortices. (pp. 179-226). New York: Plenum.

Niedermeyer, E. (1990). Alpha-like rhythmical activity of the temporal lobe. Clinical Electroencephalography, 21, 210-224.

Niedermeyer, E. (1991). The 'third rhythm': Further observations. Clinical Electroencephalography, 22, 83-96.

Niedermeyer, E. (1997). Alpha rhythms as physiological and abnormal phenomena. International Journal of Psychophysiology, 26, 31-49.

Pfurtscheller, G. (1991). Mapping procedures. In: R. Weitkunat (Ed.), Digital biosignal processing (pp. 459-480). Amsterdam: Elsevier.

Pfurtscheller, G. (1992). Event-related synchronization (ERS): An electrophysiological correlate of cortical areas at rest. Electroencephalography and Clinical Neurophysiology, 83, 62-69.

Pfurtscheller, G., \& Aranibar, A. (1977). Event-related cortical desynchronization detected by power measurements of scalp EEG. Electroencephalography and Clinical Neurophysiology, 42, 817-826.

Pfurtscheller, G., \& Berghold, A. (1989). Patterns of cortical activation during planning of voluntary movement. Electroencephalography and Clinical Neurophysiology, 72, 250-258.

Pfurtscheller, G., Zalaudek, K., \& Neuper, C. (1998). Event-related beta synchronization after wrist, finger and thumb movement. Electroencephalography and Clinical Neurophysiology, 109, 154-160.

Rösler, F. (1991). Perception or action: Some comments on preparatory negative potentials. In: C. H. M. Brunia \& M. N. Verbaten (Eds.), Event-related brain research. Electroencephalography and Clinical Neurophysiology (Suppl. 42, pp. 116-129). Amsterdam: Elsevier.

Ruchkin, D. S., Johnson, R. Jr., Mahaffey, D., \& Sutton, S. (1988). Towards a functional categorization of slow waves. Psychophysiology, 25, 339-353.

Salmelin, R., Hämäläinen, M., Kajola, M., \& Hari, R. (1995). Functional segregation of movement-related rhythmic activity in the human brain. Neuroimage, 2, 237-243.

Simons, R. F. (1984). Event-related slow brain potentials: A perspective from ANS psychophysiology. In: P. K. Ackles, J. R. Jennings, \& M. G. H. Coles (Eds.), Advances in psychophysiology. (Vol. 3, pp. $223-$ 267). Greenwich, CT: JAI Press.

Stancak, A. Jr., \& Pfurtscheller, G. (1995). Desynchronization and recovery of beta rhythms during brisk and slow self-paced finger movements in man. Neuroscience Letters, 196, 21-24.

Stancak, A. Jr., \& Pfurtscheller, G. (1996). The effects of handedness and type of movement on the contralateral preponderance of mu-rhythm desynchronization. Electroencephalography and Clinical Neurophysiology, 99, 174-182.

Stancak, A. Jr., Riml, A., \& Pfurtscheller, G. (1997). The effects of external load on movement-related changes of the sensorimotor EEG rhythms. Electroencephalography and Clinical Neurophysiology, 102, 495504.

Steriade, M., Gloor, P., Llinás, R. R., Lopes da Silva, F. H., Mesulam, M. M. (1990). Basic mechanisms of cerebral rhythmic activities. Electroencephalography and Clinical Neurophysiology, 76, 481-508.

Tiihonen, J., Hari, R., Kajola, M., Karhu, J., Ahlfors, S., \& Tissari, S. (1991). Magnetoencephalographic $10 \mathrm{~Hz}$ rhythm from the human auditory cortex. Neuroscience Letters, 129, 303-305.

Toro, C., Deuschl, G., Thatcher, R., Sato, S., Kufta, C. \& Hallet, M. (1994) Event-related desynchronization and movement-related cortical potentials on the ECoG and EEG. Electroencephalography and Clinical Neurophysiology, 93, 380-389.

Van Boxtel, G. J. M. (1994). Non-motor components of slow brain potentials. Ph.D. Thesis, Tilburg University.

Van Boxtel, G. J. M., \& Brunia, C. H. M.(1994). Motor and non-motor aspects of slow brain potentials. Biological Psychology, 38, 37-51. 
Van den Berg-Lenssen, M. M. C., Brunia, C. H. M., \& Blom, J. A. (1989). Correction of ocular artifacts in EEG's using an autoregressive model to describe the EEG; a pilot study. Electroencephalography and Clinical Neurophysiology, 73, 72-83.

Vasey, W. M., \& Thayer, J. F. (1987). The continuing problem of false positives in repeated measurements ANOVA in psychophysiology: A multivariate solution. Psychophysiology, 24, 474-486.
Wetherill, G. B. \& Levitt, H. (1965). Sequential estimation of points on a psychometric function. British Journal of Mathematical and Statistical Psychology, 18, 1-10.

(ReceIved April 10, 2000; AcCePTED May 8, 2001) 